\title{
Automatic feedback control of an Er-doped fiber laser with an intracavity loss modulator
}

\author{
F. J. Grawert, F. Ö. Ilday, D. F. Kielpinski, J. T. Gopinath, G. S. Petrich, L. A. Kolodziejski, \\ E. P. Ippen, and F. X. Kärtner \\ Department of Electrical Engineering and Computer Science and Research Laboratory of Electronics, \\ Massachusetts Institute of Technology, Cambridge, Massachusetts 02139
}

Received November 29, 2004

\begin{abstract}
Suppression of $Q$-switching instabilities with an actively controlled intracavity loss modulator is demonstrated in an Er-doped waveguide laser that is mode locked with a slow saturable absorber at repetition rates of as much as $100 \mathrm{MHz}$. By automatic gain control in the feedback loop, stable mode locking is achieved over the entire parameter range of the laser. This approach renders laser stabilization independent of the characteristics of the gain medium and intracavity power. The pulse-shaping dynamics is not affected by the presence of the intracavity loss modulator. (C) 2005 Optical Society of America

OCIS codes: $320.7090,140.4050,140.3540$.
\end{abstract}

Robustness and high repetition rates can be attained in solid-state and fiber lasers by use of saturable Bragg reflectors as starting and pulse-shaping mechanisms. ${ }^{1,2}$ In addition, their use offers the potential for future monolithic integration of the saturable absorber into the gain medium, leading to compact pulsed laser sources. ${ }^{3}$ However, mode locking with a saturable Bragg reflector can be plagued by $Q$-switching instabilities, especially in lasers with long upper-state lifetimes and high repetition rates, for which the gain dynamics occurs over a time scale much longer than the cavity round-trip time. ${ }^{4,5} \mathrm{Sev}$ eral active and passive stabilization schemes have been proposed to resolve this shortcoming. Twophoton absorption can be used as a means of passive stabilization, ${ }^{6}$ either integrated monolithically in the saturable absorber ${ }^{7}$ or as a discrete cavity element. ${ }^{8}$ This approach achieves stable pulse generation over only a narrow parameter range and typically requires tedious fine tuning of the laser cavity to prevent breakup into multiple pulses. ${ }^{4}$ In contrast, active suppression of $Q$ switching with an electronic control system decouples the $Q$-switching suppression and the pulse shaping. Consequently it allows the laser to be operated in a configuration that is more suitable for pulse shaping; i.e., the absorber does not need to be heavily saturated, allowing for shorter pulses ${ }^{4}$ and importantly avoiding the longterm material damage that occurs at high fluences. To date, active suppression of $Q$ switching has been demonstrated only by a derivative controller acting on the pump power of the laser. ${ }^{9-11}$ This technique reaches its limit in high-repetition-rate lasers with long upper-state lifetimes ${ }^{12}$ because the gain medium acting as a low-pass filter strongly dampens all fast modulations. The relaxation oscillation frequency at which the controller acts lies as much as 3 orders of magnitude above this cutoff frequency in Er- and $\mathrm{Yb}$ doped laser systems; consequently the magnitude of the control signal is reduced by the same amount. ${ }^{13}$ Thus the feedback stabilization by means of gain modulation becomes ineffective when the perturbations on the pump power are limited in amplitude, for example, by a finite current slope in the drive electronics of the pump laser diode. ${ }^{12}$

In this Letter we demonstrate suppression of $Q$ switching in a continuous-wave (cw) mode-locked laser with an intracavity loss modulator over the entire parameter range of the laser by means of automatic gain control. This approach prevents the undesired low-pass filtering of the feedback signal that is inherent in stabilization schemes that act on the pump power. Consequently it extends the well-known active stabilization schemes ${ }^{9-11}$ even to lasers with long upper-state lifetimes, large amounts of saturable loss, and high repetition rates. The primary advantage of this scheme is that it permits stability of mode locking independently of the characteristics of the gain medium and specific combinations of pump power, pulse energy, and saturable absorption.

We built a fiber laser with an Er-doped waveguide amplifier as the gain medium ${ }^{14}$ to test the proposed scheme experimentally. A schematic of the experimental setup is shown in Fig. 1. Our choice was motivated by the uncommonly long upper-state lifetime of the Er-doped medium $(7.9 \mathrm{~ms})$ and by the ease of experimentation in the fiber-coupled laser system. The laser cavity includes an Er-doped waveguide am-

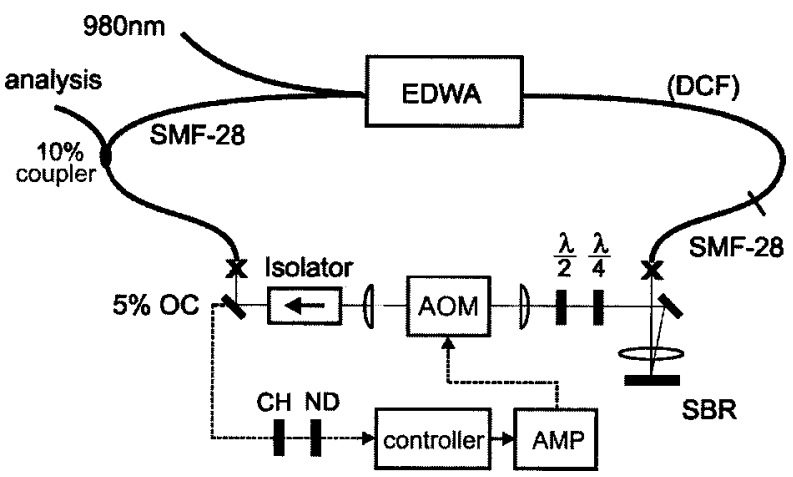

Fig. 1. Experimental setup: EDWA, Er-doped waveguide amplifier, DCF, dispersion-compensating fiber; SMFs, single-mode fibers; SBR, saturable Bragg reflector; OC, output coupler; $\mathrm{CH}$, optical chopper; ND, neutral-density filter; AMP, driver and amplifier. 
plifier, which is pumped by a $980-\mathrm{nm}$ laser diode with as much as $300 \mathrm{~mW}$ of pump power. The lead fibers of the Er-doped waveguide amplifier and the $10 \%$ output coupler are SMF-28, with a total length of $5.4 \mathrm{~m}$. A 0.9-m-long segment of dispersion-compensating fiber $\left(+90,000 \mathrm{fs}^{2}\right)$ keeps the net cavity dispersion close to zero $\left(-20,000 \mathrm{fs}^{2}\right)$ to permit short and energetic pulses. ${ }^{14}$ The spot size on the InGaAs/InP-based stable Bragg reflector, grown upon a GaAs/AlAs reflector, is $13 \mu \mathrm{m}$. Pump-probe measurements determined the saturable loss to be $16 \%$ at $1540 \mathrm{~nm}$, with a recovery time of $43 \mathrm{ps}$. A half-wave and a quarterwave plate are placed to convert the polarization of the light rotated in the fiber section into $p$ polarization, in alignment with the isolator and the acoustooptic modulator (AOM) that serves as an intracavity loss modulator. The isolator provides for unidirectional propagation. Linearly polarized light is coupled back into the fiber to prevent accidental amplitude modulation owing to nonlinear polarization rotation. The corresponding repetition rate of the cavity is $29 \mathrm{MHz}$. Higher repetition rates of 39 and $101 \mathrm{MHz}$ were also studied and are discussed below.

The feedback circuit constitutes a proportional controller ( $\mathrm{P}$ controller) rather than the derivative controller that was used previously. ${ }^{9-11}$ The electronics consists of a photodiode with a $70-\mathrm{MHz}$ transimpedance amplifier, a limiting amplifier, an automatic gain control circuit, a bandpass filter, and a gain stage with adjustable dc bias. The signal of the controller feeds a commercial AOM driver whose output is amplified by a rf amplifier to produce a maximum modulation depth of $45 \%$. The propagation delay in the AOM is $\sim 200 \mathrm{~ns}$. The loss introduced by the AOM is proportional to the cosine squared of the applied drive voltage. To reduce this nonlinearity, the AOM is slightly biased and permanently introduces a small amount of loss, such that it can both increase and decrease intracavity losses. The controller incorporates a bandpass filter to block both dc signals and higher frequencies to preserve this operating point regardless of either the pump power or the mode-locking state $[Q$-switched mode locking (QSML) or cw mode locking] and to prevent the circuit from interfering with the individual pulses at the repetition rate. A steep roll-off at $6 \mathrm{MHz}$ is implemented by a fourthorder Bessel filter that suppresses the repetition rate and its harmonics by at least $40 \mathrm{~dB}$ while it allows for maximum controller bandwidth. The gain of the $\mathrm{P}$ controller is adjusted with a variable neutral-density filter in the beam incident upon the photodiode, whereas a fast limiting amplifier prevents oversaturation and subsequent slow recovery from a $Q$-switch cycle by keeping the output signal of the controller within the limits of the AOM driver.

The control experiment is given by the observation that without feedback stabilization the laser exhibits only either pure $Q$ switching or QSML at pump powers ranging from lasing threshold to maximum available power. This behavior is evident from the rf spectrum [Fig. 2(a), inset]. However, when the feedback controller is engaged, $Q$-switch sidebands are suppressed by $38 \mathrm{~dB}$, resulting in a clean rf spectrum
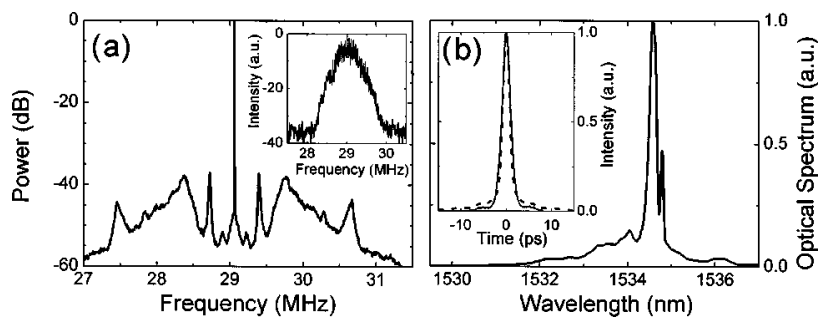

Fig. 2. Characteristics of the stabilized laser at $29 \mathrm{MHz}$. (a) rf spectrum in stabilized mode-locked and (inset) QSML operation. (b) Optical spectrum; inset, autocorrelation (solid curve) and autocorrelation of the zero-phase Fourier transform of the optical spectrum (dashed).
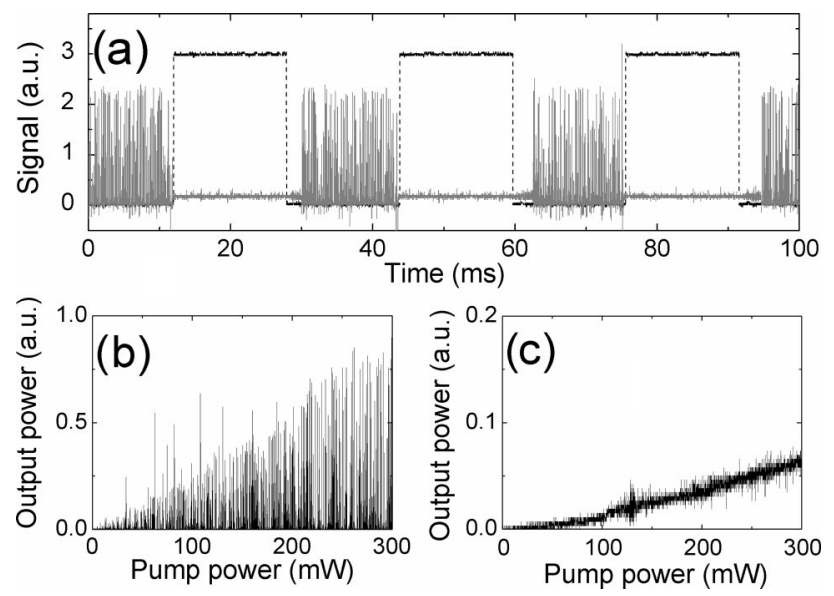

Fig. 3. (a) When the controller is blocked by an optical chopper (darker, dashed lines), the laser (lighter, solid curves) $Q$ switches, whereas it is cw mode locked when the controller is effective. (b) During ramp-up of the current, the laser $Q$-switches for all pump power levels without the controller, while (c) the instabilities are suppressed by the feedback.

with no further sidebands at higher frequencies. The stabilized laser shows a smooth optical spectrum centered at $1534.5 \mathrm{~nm}$ [Fig. 2(b)]. The FWHM pulse duration is inferred from the autocorrelation measurements to be $1.5 \mathrm{ps}$, assuming a Gaussian pulse shape. The measured pulse duration exceeds that extracted from the zero-phase Fourier transform by 20\% [Fig. 2 (b), inset]. The output power obtained from the $10 \%$ coupler port is $0.6 \mathrm{~mW}(0.3 \mathrm{~mW}$ from the $5 \%$ output coupler), corresponding to an intracavity pulse energy of $210 \mathrm{pJ}$, for a pump power of $200 \mathrm{~mW}$. The sideband suppression is limited only by the available control loop bandwidth, which is dominated by the phase lag introduced by the propagation delay in the AOM. For higher values of feedback gain, the feedback loop becomes unstable and begins to oscillate.

Robust and repeated suppression of $Q$ switching, as well as self-starting operation of the laser, is demonstrated by periodic turning of the control loop on and off by blocking the input photodetector of the control circuit periodically with a chopper [Fig. 3(a)]. When it is blocked, the controller is inactive and consequently the laser $Q$ switches. When the controller is active, suppression is attained reliably within a few $Q$-switch cycles. This ability of the controller to 
switch the laser from irregular QSML to a stable cwmode-locked state was observed for all repetition rates and power levels. Another goal of active stabilization is attained by use of automatic gain control in the feedback circuit to allow the controller to stabilize the laser over the entire parameter range of the laser for all levels of pump power at a repetition rate of $29 \mathrm{MHz}$. Without feedback stabilization the laser exhibits $Q$ switching and QSML for all power levels when the pump power of the laser is ramped slowly from zero to $300 \mathrm{~mW}$ [Fig. 3(b)]. In contrast, with the controller activated, stable $\mathrm{cw}$ and cw-mode-locking operation is attained over the entire parameter range [Fig. 3(c)]. The automatic gain control circuit keeps the time-varying output of the controller at a constant level regardless of the optical power incident upon the photodiode of the controller.

Stabilization at higher repetition rates of the lasers was also investigated. We decreased the fiber lengths to attain the desirable repetition rate. With the single-mode fiber's length set to $4.6 \mathrm{~m}$ and the dispersion-compensating fiber removed from the cavity, the repetition rate increased to $39 \mathrm{MHz}$ $\left(-90,000-\mathrm{fs}^{2}\right.$ net dispersion). At $150-\mathrm{mW}$ pump power a FWHM pulse width of 4.8 ps (12\% above the Fourier limit) was observed in autocorrelation measurements and an output power of $0.96 \mathrm{~mW}$ was measured at the $10 \%$ coupler, corresponding to a pulse energy of $250 \mathrm{pJ}$. The noise level was suppressed as much as $62 \mathrm{~dB}$ [Fig. 4(a)], and the pulse train of the stabilized laser recorded with a $150-\mathrm{MHz}$ analog oscilloscope and a $2-\mathrm{GHz}$ detector showed stable and regular mode locking [Fig. 4(a), inset]. At even higher repetition rates the phase lag of the AOM renders stabilization increasingly difficult. The repetition rate was increased to $101 \mathrm{MHz}$ by reduction of the single-mode fiber to $1.4 \mathrm{~m}\left(-10,000-\mathrm{fs}^{2}\right.$ net dispersion). The $150-\mathrm{mW}$ pump power produced an output power of $0.9 \mathrm{~mW}$ (90-pJ pulse energy) from the $10 \%$ coupler. Stabilized operation is possible only for a narrow range of gain and offset values in the feedback loop. The laser can be stabilized for different values of pump power by manual fine tuning of the control circuit parameters; suppression of the noise level to $-42 \mathrm{~dB}$ was achieved [Fig. 4(b)]. However, automated stabilization over the entire range of pump power is impossible. In this state, because of the lower intracavity pulse energy and consequently smaller saturation of the absorber, longer pulses are generated.

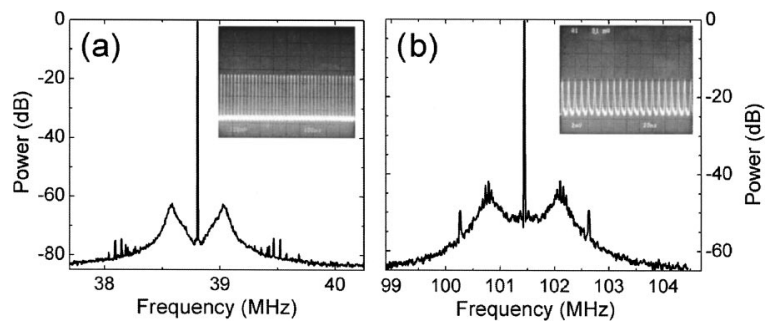

Fig. 4. rf spectra with an activated controller and a pulse train recorded with a $150-\mathrm{MHz}$ analog oscilloscope (inset): (a) $39-\mathrm{MHz}$ repetition rate, (b) $101-\mathrm{MHz}$ repetition rate.
These results show that the time-dependent loss introduced by the intracavity modulator does not adversely affect mode locking. Intuitively, one can understand this by recalling that (i) the effects of the controller and the pulse-shaping mechanism occur on different time scales and (ii) once stabilized, the losscontrol mechanism (the AOM) practically exerts a negligible modulation.

In conclusion, we have demonstrated suppression of $Q$-switching instability in a laser system with millisecond gain recovery time and a strong saturable absorber with $16 \%$ saturable absorption by direct feedback control of the net intracavity loss. In contrast to modulation of the gain through pump power, this scheme is independent of the properties of the gain medium and is applicable to systems with strong saturable absorption. In the future, the demonstrated feedback scheme can be applied to the stabilization of gigahertz-repetition-rate lasers with fast intracavity loss modulators, which should permit the use of saturable absorbers in an operating point optimized for pulse shaping rather than for stability. ${ }^{5}$

The authors thank Frank W. Wise for the loan of the Er-doped waveguide amplifier and Bookham Technology plc. for providing the $980-\mathrm{nm}$ pump diode for this experiment. This research was supported by the National Science Foundation under grants ECS0322740, ONR-N00014-02-1-0717, DARPA W911NF04-1-0431, and AFOSR FA9550-04-1-0011. F. J. Grawert's e-mail address is felixg@mit.edu.

\section{References}

1. S. Tsuda, W. H. Knox, S. T. Cundiff, W. Y. Jan, and J. E. Cunningham, IEEE J. Sel. Top. Quantum Electron. 2, 454 (1996).

2. S. C. Zeller, F. Krausz, G. J. Spuehler, R. Paschotta, M. Golling, D. G. Ebling, K. J. Weingarten, and U. Keller, Electron. Lett. 40, 875 (2004).

3. F. J. Grawert, J. T. Gopinath, F. O. Ilday, H. Shen, E. P. Ippen, F. X. Kärtner, S. Akiyama, J. Liu, K. Wada, and L. C. Kimerling, Opt. Lett. 30, 329 (2005).

4. F. X. Kärtner, I. D. Jung, and U. Keller, IEEE J. Sel. Top. Quantum Electron. 2, 540 (1996).

5. C. Hönninger, R. Paschotta, F. Morier-Genoud, M. Moser, and U. Keller, J. Opt. Soc. Am. B 16, 46 (1999).

6. T. R. Schibli, E. R. Thoen, F. X. Kärtner, and E. P. Ippen, Appl. Phys. B 70, 41 (2000).

7. E. R. Thoen, E. M. Koontz, M. Joschko, P. Langlois, T. R. Schibli, F. X. Kärtner, E. P. Ippen, and L. A. Kolodziejski, Appl. Phys. Lett. 74, 3927 (1999).

8. P. Cerný, G. Valentine, D. Burns, and K. McEwan, Opt. Lett. 29, 1387 (2004).

9. S. Bielawski, M. Bouazaoui, D. Derozier, and P. Glorieux, Phys. Rev. A 47, 3276 (1993).

10. N. Joly and S. Bielawski, Opt. Lett. 26, 692 (2001).

11. T. R. Schibli, U. Morgner, and F. X. Kärtner, Opt. Lett. 26, 148 (2001).

12. N. Joly and S. Bielawski, Opt. Commun. 220, 171 (2003).

13. C. Svelto, S. Taccheo, M. Marano, G. Sorbello, and P. Laporta, Electron. Lett. 36, 1623 (2000).

14. D. Barbier, M. Rattay, F. S. Andre, G. Clauss, M. Trouillon, A. Kevorkian, J.-M. P. Delavaux, and E. Murphy, IEEE Photonics Technol. Lett. 9, 315 (1997). 